\title{
An Optimization and Selection Method for Planning Projects of MV Distribution Network Considering Categorization of Project Attributes
}

\author{
YANG Weihong ${ }^{1, ~ a ~, ~ L I U ~ H o n g ~}{ }^{2, b}$, CUI Wenting ${ }^{2, c}$, YANG Zan ${ }^{2}$ and WANG \\ Xuyang ${ }^{1}$ \\ ${ }^{1}$ State Power Economic Research Institute, Beijing 102209, China \\ ${ }^{2}$ Key Laboratory of Smart Grid of Ministry of Education (Tianjin University), Tianjin 300072, China \\ a yangweihong@chinasperi.sgcc.com.cn, ${ }^{\mathrm{b}}$ liuhong@tju.edu.cn, ${ }^{\mathrm{c}}$ cwt1102@126.com
}

Keywords: planning projects; project attributes; evaluation indicators; project optimization and selection.

Abstract. In order to solve the lack in specific evaluation and comparison standards on planning projects of distribution networks, which are widely spread in Chinese grid companies, an optimization and selection method for planning projects of MV distribution networks considering categorization of project attributes is proposed. Firstly, an evaluation indicator system for planning projects of distribution networks is established, and corresponding indicator calculation method, weights and determination method on grading standard are presented. Next, the attributes of planning projects in distribution networks are categorized, and the mapping relation between attributes and evaluation indicators of planning projects is constructed, based on which the determination method on the significances of project attributes is proposed. Then, an optimization and selection model for planning projects of distribution networks is constructed, and an optimization and selection method of planning projects in distribution networks considering investment limit, project attributes and project grading is established. Finally, the results from typical case analysis have verified the effectiveness of the research achievements in this paper.

\section{Introduction}

In recent years, the electric power loads in China have grown at a steady pace. As a key link to secure high-quality and high-reliability power supply for end users, the distribution networks are featured by large-scaled investments, which tend to increase year by year on the whole. In this circumstance, the investment efficiency of distribution networks has become a key issue focused by grid companies. However, the imbalance between investment demand and investment capability is widely spread in current Chinese grid companies. The lack of specific evaluation and comparison standards on planning projects of distribution networks leads to difficulties in determining investment targets in distribution networks and arranging investment projects. As a result, there is massive room for improvement on investment efficiency of distribution networks, and it is of urgent need to construct scientific and practical project optimization and selection method in distribution networks in order to realize scientific arrangement of investment projects, rationally master investment time sequence, steadily improve investment efficiency in distribution networks as well as avoid blind investments and repeated construction.

To be specific, the current related research literature can be basically divided into two categories. The first category is to study the project sequencing, optimization \& selection and investment decisions. In [1], the optimization sequencing model for planning projects in distribution networks was proposed and can be applied in determining the project construction time sequence, but the results of project optimization and selection cannot be determined. Then, [2, 3] presented the sequencing method for project optimization and selection from different perspectives but have not considered the constraint of investment limit, and thus its application is limited in regions with finite funds. In [4, 5], the optimization method for project decisions was presented from different perspectives respectively and able to obtain the optimal combination of distribution network projects under the constraint of investment limit, but the influence which categorization of planning project 
attributes has on the sequencing of optimization and selection was not considered. The second category is to propose the evaluation and comparison method on planning schemes of distribution networks from different perspectives ${ }^{[6-8]}$ and place particular emphasis on the comparison among different planning schemes but has not involved the study on project optimization and selection in the specific planning schemes.

On the basis of above analysis, an optimization and selection method for planning projects in distribution networks considering categorization of project attributes is proposed in this paper. Firstly, the evaluation indicator system for planning projects in distribution networks is established, and corresponding indicator calculation method, weights and determination method on grading standard are presented; next, the analysis method on the significances of different planning project attributes in distribution networks is put forward in order to reflect the implementation urgency of different planning projects; then, an optimization and selection model for planning projects in distribution networks is established, which comprehensively considers the investment limit of distribution networks, significances of project attributes and project evaluation indicator system for the purpose of determining the optimization and selection results of planning projects in distribution networks; finally, a typical case analysis is carried out.

It needs to be noted that the optimization and selection method for planning projects in distribution networks proposed in this paper is applicable to MV distribution networks. Therefore, the distribution networks mentioned below refer in particular to MV distribution networks.

\section{Evaluation Indicator System for Planning Projects in Distribution Networks}

Indicator system. The purpose of implementing planning projects in distribution networks is to improve the overall performance of distribution networks and enhance the operating effect of current power grids. In addition, the implementation of planning projects in distribution networks is mainly to solve the problems in power grid structure, power equipment level and power supply capability of distribution systems. Consequently, the evaluation indicator system for planning projects in distribution networks can be constructed from above three aspects as shown in Fig. 1 in order to reflect the implementation effect of planning projects in distribution networks.

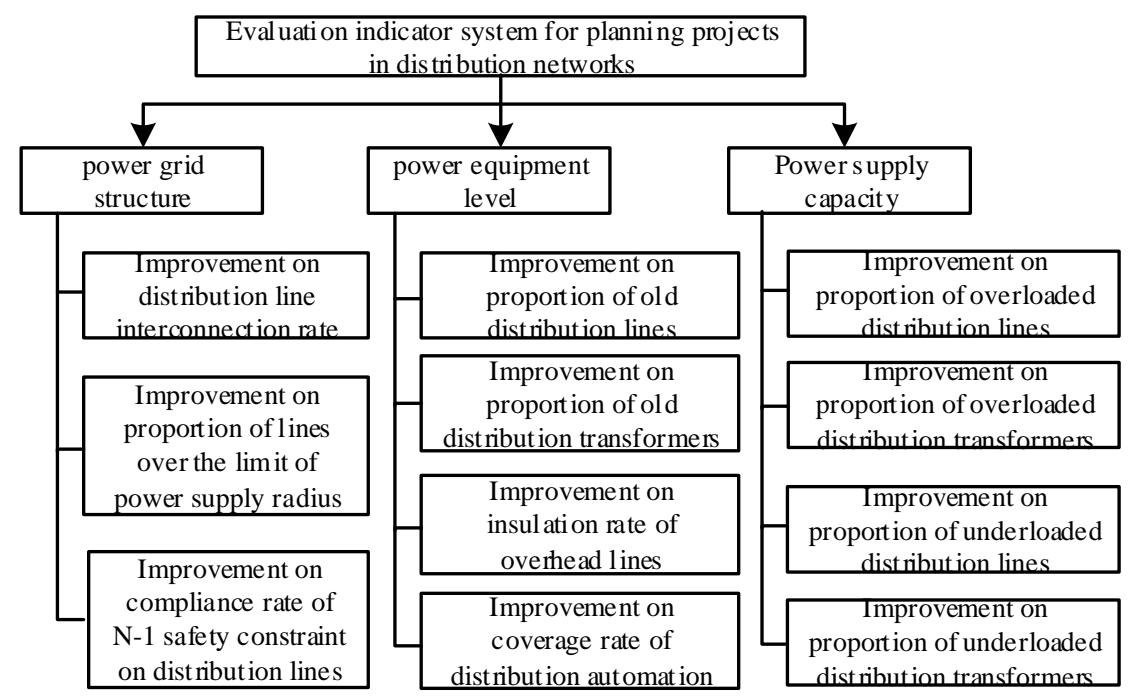

Fig.1 Evaluation indicator system for planning projects in distribution networks

Indicator calculation method. It can be seen from Fig.1 that the evaluation indicators for planning projects in distribution networks refer to the improvement on corresponding operating state indicators. Thus, they emphasize on embodying the improvement on operating states of distribution networks and can be calculated through the change in operating states after the projects are implemented. However, considering that the dimensions of different operating state indicators vary, the change in operating states after the projects are implemented will differ in dimensions as well. 
Therefore, in order to convert the indicators into a unified dimension and facilitate evaluation process[9], a normalized coefficient is applied to make all the indicators dimensionless. The normalized coefficient is defined as the reciprocal of improvable space of the indicators before the projects are implemented while the improvable space refers to the difference between the indicator value before the projects are implemented and the ideal value. The indicator calculation method is stated as follows.

When the operating state indicator is an efficiency-based indicator ${ }^{[10]}$, the ideal indicator value is 100\%. Assuming that $N_{1}$ and $N_{2}$ are the indicator values before and after the projects are implemented respectively, the normalized coefficient is defined as $\left(100 \%-N_{1}\right)^{-1}$. The actual improved indicator value is related to change in the indicator value. The calculation for the value of evaluation indicator $x$ is given in Eq. 1 .

When the operating state indicator is a cost-based indicator ${ }^{[10]}$, the ideal indicator value is 0 . Assuming that $N_{1}^{\prime}$ and $N_{2}^{\prime}$ are the indicator values before and after the projects are implemented respectively, the normalized coefficient is defined as $N_{1}^{-1}$. In the same way, the calculation for evaluation indicator $x^{\prime}$ is expressed in Eq. 2:

$$
\begin{aligned}
& x=\left\{\begin{array}{l}
\frac{N_{2}-N_{1}}{100 \%-N_{1}} \times 100 \%, \text { when } N_{1} \leqslant N_{2} \\
0, \text { when } N_{1}>N_{2}
\end{array}\right. \\
& x^{\prime}=\left\{\begin{array}{l}
\frac{N_{1}^{\prime}-N_{2}^{\prime}}{N_{1}^{\prime}} \times 100 \%, \text { when } N_{1}^{\prime} \geqslant N_{2}^{\prime} \\
0, \text { when } N_{1}^{\prime}<N_{2}^{\prime}
\end{array}\right.
\end{aligned}
$$

Based on above dimensionless processing, all the evaluation indicators are converted into the same dimension and are efficiency-based indicators, which give them comparability.

Determination method on indicator weights. In combination of analytic hierarchy process, the weights of different evaluation indicators are determined through theoretical calculation based on the difference between the current value and the ideal value of each operating state indicator.

Firstly, the weights of evaluation indicators on planning projects in distribution networks need to embody the urgency to improve corresponding operating state indicators, and the difference between the actual value and the ideal value of operating state indicators can reflect the urgency to improve the corresponding indicators. A greater difference means a more urgent need to improve the indicators and thus a higher indicator weight.

Next, the relative indicator significances are obtained on the basis of the "difference between the actual value and the ideal value" of each two operating state indicators; a greater difference corresponds to a higher scaled value of the indicator. In the judgment matrix, the relation between the relative significance of two indicators and the "difference between the actual value and the ideal value" of each two operating state indicators is given as:

$$
\beta_{i j}=\left\lfloor\frac{9}{\Delta M_{\max }} \times \frac{M_{i}}{M_{j}}\right\rfloor
$$

Where, $\beta_{i j}$ represents the relative significance between indicator $i$ and indicator $j$, ranges between 1 and 9 , and is rounded up to an integer. Assuming that the "difference between the actual value and the ideal value" of indicator $i, M_{i}$ is larger than the "difference between the actual value and the ideal value" of indicator $j, M_{j}, \Delta M_{\max }$ is the ratio between the maximum difference and the minimum difference.

Based on Eq. 3, the relative significance of each two indicators can be calculated and corresponding judgment matrix is constructed. In combination of analytic hierarchy process, the weights of evaluation indicators for planning projects in distribution networks can be obtained. 
Determination method on indicator grading standard. The grading standard refers to the way to convert all the raw data into normalized format which can be directly compared through a certain scale system. In this paper, the hundred-mark system is adopted.

It can be known from the definitions and calculation methods of different evaluation indicators that the ideal value of all the indicators is $100 \%$. In order to formulate a rational grading standard for each evaluation indicator, it is assumed that the corresponding relation between indicator values and grades is linear, the grading standard is given as:

$$
y=100 x
$$

Where, $x$ is the indicator value, and $y$ is the indicator grade.

\section{Categorization of Project Attributes and Determination of Significances}

Definitions and categorization of project attributes. Project attributes refer to the measures to solve current problems in distribution networks, and the planning projects can be categorized according to project attributes. In combination of the goal of implementing planning projects in distribution networks, the planning projects can be categorized in to 8 categories, including satisfaction of power supply demand for newly-added loads, solution to power supply bottleneck, solution to power equipment overloading problem, elimination of equipment safety hazards, auxiliary substation distribution lines, improvement on distribution network structure, renovation of distribution transformers with high loss rate, and construction of distribution automation.

Mapping relation between project attributes and evaluation indicators. It can be known from the definitions of project attributes that the projects with the same attributes can improve multiple operating state indicators while the same operating state indicator can be improved by multiple projects with different attributes. Table 1 shows the mapping relation between 7 categories of project attributes and the operating state indicators.

Table 1 Mapping relation between project attributes and evaluation indicators

\begin{tabular}{|c|c|}
\hline Project attributes & Set of coupling indicators \\
\hline $\begin{array}{l}\text { Satisfaction of power supply demand for newly-added } \\
\text { loads }\end{array}$ & Improvement on compliance rate of $\mathrm{N}-1$ safety constraint on distribution lines \\
\hline Solution to power supply bottleneck & $\begin{array}{l}\text { Improvement on proportion of overloaded distribution lines } \\
\text { Improvement on proportion of overloaded distribution transformers }\end{array}$ \\
\hline Solution to equipment overloading problem & $\begin{array}{l}\text { Improvement on proportion of overloaded distribution lines } \\
\text { Improvement on proportion of overloaded distribution transformers }\end{array}$ \\
\hline Elimination of equipment safety hazards & $\begin{array}{l}\text { Improvement on proportion of old distribution lines } \\
\text { Improvement on proportion of old distribution transformers } \\
\text { Improvement on insulation rate of overhead lines }\end{array}$ \\
\hline Auxiliary substation distribution lines & $\begin{array}{l}\text { Improvement on compliance rate of } \mathrm{N}-1 \text { safety constraint on distribution lines } \\
\text { Improvement on proportion of lines over the limit of power supply radius }\end{array}$ \\
\hline Improvement on distribution network structure & $\begin{array}{l}\text { Improvement on compliance rate of } \mathrm{N}-1 \text { safety constraint on distribution lines } \\
\text { Improvement on distribution line interconnection rate } \\
\text { Improvement on proportion of underloaded distribution lines } \\
\text { Improvement on proportion of underloaded distribution transformers }\end{array}$ \\
\hline $\begin{array}{l}\text { Renovation of distribution transformers with high loss } \\
\text { rate }\end{array}$ & Improvement on proportion of old distribution transformers \\
\hline Construction of distribution automation & Improvement on coverage rate of distribution automation \\
\hline
\end{tabular}

Determination of project attribute significances. It can be known from the definitions of project attributes that the projects with the same attributes can improve multiple operating state indicators while the same operating state indicator can be improved by multiple projects with different attributes. Table 1 shows the mapping relation between 7 categories of project attributes and the operating state indicators.

The implementation of one certain project attribute is more beneficial to improve the distribution networks and the corresponding implementation significance is higher if the project can solve more current problems in distribution networks and improve more evaluation indicators with higher weights. Therefore, the significance of project attributes is given as: 


$$
H_{k}=\sum_{i=1}^{n} \omega_{i k}
$$

Where, the significance of projects in $k t h$ category, $H_{k}$, is the sum of weights of improvable indicators in $k t h$ category, $\omega_{i k}$ is the weight of ith evaluation indicator which can be improved by projects in kth category.

\section{Optimization and Selection of Planning Projects in Distribution Networks}

Initial project grading. Based on the indicator calculation method, determination method of indicator weight and indicator grading standard for planning projects in distribution networks, the initial project grading is given as:

$$
G=\sum_{j=1}^{l} y_{j} \omega_{j}
$$

Where, $G$ is the initial grade of one planning project; $j$ is the number of evaluation indicators and there are $l$ evaluation indicators in total; $y_{j}$ is the grade of $j$ th evaluation indicator; $\omega_{j}$ is the weight of $j$ th evaluation indicator.

Revised project grading. In combination of the significances of project attributes, the initial project grading is revised. If the project attribute is more significant, the urgency to implement this category of projects is higher and the corresponding project grade is higher. The revised project grade is given as:

$$
G^{\prime}=H \times G
$$

Where, $G^{\prime}$ is the revised grade of one planning project, $H$ is the significance of project attribute corresponding to this planning project and $G$ is the initial grade of planning project.

Project optimization and selection model. The purpose of planning project optimization and selection is to obtain the set of projects which can improve the distribution networks to maximum in combination of the revised grades of planning projects under the condition that the investment limit of planning projects is satisfied. The optimization and selection sequencing model for planning projects is given as:

$$
\left\{\begin{array}{l}
F=\max \left(\sum_{i=1}^{m} G^{\prime}\left(P_{i}\right)\right) \\
\text { s.t. } \\
P_{i} \in P_{\text {total }} \\
\sum_{i=1}^{m} C\left(P_{i}\right) \leq \mathrm{C}_{\max }
\end{array}\right.
$$

Where, $F$ is the objective function of the optimization and selection sequencing model for planning projects; $G^{\prime}\left(P_{i}\right)$ is the revised grade of $i t h$ planning project and $m$ is the number of planning projects; $P_{\text {total }}$ is the set of all the planning projects; $C\left(P_{i}\right)$ is the investment needed by ith planning project; $C_{\max }$ is the investment limit for grid companies.

\section{Case Study}

In the typical case of County B, City A, the optimization and selection of planning projects in distribution networks in 2016 are carried out. In County B, the planned total investment in distribution networks in 2016 is RMB 53 million yuan. In addition, 163 projects in total are submitted and the total investment reaches RMB 73.35 million yuan.

Survey on indicator data and calculation of indicator weights. The current data of distribution networks in County B in 2016 are surveyed; in combination of the calculation method on evaluation 
indicator weights for planning projects proposed in Chapter 1.3, the weights of different indicators can be obtained in Table 2.

Table 2 Operating state indicator values and evaluation indicator weights of planning projects in distribution networks in County B

\begin{tabular}{|c|c|c|c|c|}
\hline Operating state indicators & Unit & Current values & Ideal values & $\begin{array}{c}\text { Corresponding evaluation } \\
\text { indicator weights }\end{array}$ \\
\hline Distribution line interconnection rate & $\%$ & 36.1 & 100 & 0.0935 \\
\hline Proportion of distribution lines over the limit of power supply radius & $\%$ & 25.3 & 0 & 0.0916 \\
\hline Compliance rate with $\mathrm{N}-1$ safety constraint on distribution lines & $\%$ & 31.8 & 100 & 0.0982 \\
\hline Proportion of old distribution lines & $\%$ & 2.9 & 0 & 0.0618 \\
\hline Proportion of old distribution transformers & $\%$ & 4.2 & 0 & 0.0635 \\
\hline Insulation rate of overhead lines & $\%$ & 33.9 & 100 & 0.1052 \\
\hline Coverage rate of distribution automation & $\%$ & 14.7 & 100 & 0.1841 \\
\hline Proportion of overloaded distribution lines & $\%$ & 5.2 & 0 & 0.0819 \\
\hline Proportion of overloaded distribution transformers & $\%$ & 7.3 & 0 & 0.0833 \\
\hline Proportion of underloaded distribution lines & $\%$ & 4.6 & 0 & 0.0708 \\
\hline Proportion of underloaded distribution transformers & $\%$ & 3.9 & 0 & 0.0661 \\
\hline
\end{tabular}

Calculation of project attribute significances. The calculation method on attribute significances of planning projects put forward in Chapter 2 is adopted to calculate the significances of different project attributes. Table 3 shows the calculation results.

Table 3 Calculation results of attribute significances of planning projects in distribution networks in County B

\begin{tabular}{cc}
\hline Project attributes & Calculation results of significances \\
\hline Satisfaction of power supply demand for newly-added loads & 0.0982 \\
Solution to power supply bottleneck & 0.1652 \\
Solution to equipment overloading problem & 0.1652 \\
Elimination of equipment safety hazards & 0.2305 \\
Auxiliary substation distribution lines & 0.1898 \\
Improvement on distribution network structure & 0.3286 \\
Renovation of distribution transformers with high loss rate & 0.0635 \\
Construction of distribution automation & 0.1841 \\
\hline
\end{tabular}

Planning project optimization and selection. Firstly, the calculation method described in Chapter 1.2 is applied to calculate different planning evaluation indicator values after each project is implemented;

Then, the determination method presented in Chapter 1.4 is applied to determine the grade of each one evaluation indicator, and the initial grade of each project is calculated in combination of indicator weights;

Then, in combination of the significances of project attributes, the revised grade of each project is calculated.

Table 4 shows the calculation results for some projects in County B.

Table 4 Planning evaluation indicator values in distribution networks in County B

\begin{tabular}{cccccc}
\hline Project number & Project 1 & Project 2 & Project 3 & Project 4 & Project 5 \\
\hline Indicator 01 & 0.0767 & 0 & 0 & 0.0594 & 0.0153 \\
Indicator 02 & 0.3921 & 0.4582 & 0 & 0 & 0.1194 \\
$\ldots$ & & & & & \\
Indicator 11 & 0.1130 & 0 & 0.5194 & 0.2841 & 0 \\
Initial grade & 8.71 & 7.92 & 5.04 & 5.19 & 6.22 \\
Revised grade & 2.862 & 0.778 & 1.656 & 0.985 & 1.181 \\
\hline
\end{tabular}

Finally, comprehensively considering the planned total investment in distribution networks and the revised project grades in County $\mathrm{B}$, the project optimization and selection model is applied to determine the ultimate optimization and selection result for distribution networks in County $\mathrm{B}$. The optimized results includes 129 projects in total with a total investment of RMB 50.81 million yuan. 


\section{Conclusion}

In this paper, a practical optimization and selection method for planning projects in MV distribution networks considering categorization of project attributes is proposed. This method can be applied in investment management of distribution networks by grid companies to formulate more scientific investment strategies in distribution networks. In addition, it can secure reasonable investment inputs into distribution networks, improve the investment efficiency of distribution networks, promote both economic and social benefits for grid companies, and enhance their sustainable development.

\section{Acknowledgements}

This work was supported by Technology Project of State Grid Corporation of China (Project Name: Study on Investment Assessment and Optimization of Distribution Network Planning Project based on Reliability).

\section{References}

[1] Li Can-bing, FU Mei-ping, LIANG Jin-zhao, etc: Optimization sequencing for grid construction projects based on evaluation of network coordination, Power System Protection and Control, Vol. 38 (2010), p. 112-120

[2] Song Lin-li, YANG Jun, ZHOU Bo-wen, etc: Assistant decision making scheme for construction projects of power network, Electric Power Automation Equipment, Vol. 33 (2013), p. 64-69.

[3] Peng Ding-wu: Zhongshan Power Grid Investment Optimization Model and Its Application, [M.S. thesis]. South China University of Technology, Guangzhou,China (2013).

[4] Ge Shao-yun, XU Don-gxing, LIU Hong, etc: A two-stage integrated decision optimization method based on the established project library, Power System Protection and Control, Vol. 38 (2010), p. 118-128.

[5] Yu Ying: Optimization and Implementation solution of Grid Construction Investment, [M.S. thesis]. North China Electric Power University, Beijing,China (2009).

[6] Cui Wei, Cui Yingying, Yang Haifeng, etc: Comprehensive Decision-Making of Transmission Network Planning Based on Entropy Weight and Grey Relational Analysis, Electric Power Construction, Vol. 34 (2013), p. 112-117.

[7] Luo Yi, Li Yu-long: Investment Decision Model in Special Fields for Power Grid, Power System Technology, Vol. 37 (2013), p. 77-81.

[8] Nie Hong-zhan, LV Pan, Qiao Yi, etc: Comprehensive Fuzzy Evaluation for Transmission Network Planning Scheme Based on Entropy Weight Method, Power System Technology, Vol. 33 (2009), p. 60-64

[9] Guo Ya-jun, Lv Ping-tao, etc: Character Analysis of Linear Dimensionless Methods, Statistical Research, Vol. 25 (2008), p. 26-30

[10] Hu Yong-hong: Understanding and Discussion of Some Problems in Statistical Synthesis Evaluation, Statistical Research, Vol. 29 (2012), p. 26-30 